\title{
Cloud Driven Variability on Young Brown Dwarfs and Giant Exoplanets
}

\author{
Beth Biller ${ }^{1}$ \\ ${ }^{1}$ Institute for Astronomy, University of Edinburgh \\ email: bb@roe.ac.uk
}

\begin{abstract}
Variability has now been robustly observed in a range of $\mathrm{L}$ and $\mathrm{T}$ type field brown dwarfs, primarily at near-IR and mid-IR wavelengths. The probable cause of this variability is surface inhomogeneities in the clouds of these objects, causing a semi-periodic variability signal when combined with the rotational modulation from the 3-12 hour period expected for these objects. Variability at similar or even higher amplitudes may be expected for young brown dwarfs and giant exoplanets, which share similar $T_{\text {eff }}$ as field brown dwarfs, but have considerably lower surface gravities. Variability studies of these objects relative to old field objects is then a direct probe of the effects of surface gravity on atmospheric structure. Here I discuss ongoing efforts to detect variability from these young objects, both for free-floating objects and companions to stars, including preliminary results from an ongoing survey of young, low surface gravity objects with NTT SOFI.
\end{abstract}

Keywords. infrared: planetary systems, (stars:) brown dwarfs, planets and satellites: atmospheres

\section{Introduction}

Numerous young exoplanets have been directly imaged in the infrared (c.f. among others, Marois et al. 2008; Marois et al. 2010; Lagrange et al. 2010; Rameau et al. 2013a) Initial observations of directly imaged planets have yielded some surprises. Young directly imaged exoplanets were expected to share similar atmospheric properties with the wellstudied population of brown dwarfs, which have comparable temperatures albeit higher masses. However, most young directly imaged exoplanets are much redder in the near-IR than their brown dwarf counterparts at similar $\mathrm{T}_{\text {eff }}$. In fact, only two directly imaged exoplanets (GJ 504b, Kuzuhara et al. 2013; Janson et al. 2013, HD 95086b, Rameau et al. 2013a; Rameau et al. 2013b) to date possesses the methane absorption feature robustly observed in brown dwarfs with similar $\mathrm{T}_{\text {eff }}$. This discrepancy may be explained by the persistence of dusty clouds at lower temperatures as well as non-equilibrium chemistry (e.g., Barman et al. 2011; Marley et al. 2012), likely to result from the lower surface gravity of these objects. Furthermore, patchy cloud coverage may be required to model observations across a wide wavelength range (e.g., HR 8799b; Bowler et al. 2010; Skemer et al. 2012). Like their higher mass brown dwarf counterparts (Zapatero Osorio et al. 2006), planets are expected to rotate on hour to day timescales. Thus, a key probe of cloud properties in exoplanet atmospheres is time-variability (brightness as a function of phase), which is sensitive to the spatial distribution of condensates as the planet rotates. 


\section{Conditions for Variability}

Detectable rotationally modulated variability in a specific class of object requires the presence of: 1) relatively short rotation periods (i.e. observable on the timescales of a night or two) and 2) surface inhomogeneities.

It is already clear that field brown dwarfs possess quite short rotational periods. (Zapatero Osorio et al. 2006) obtained projected rotational velocities (vsini) for a sample of 19 M6.5-T8 very low mass stars and brown dwarfs using KECK NIRSPEC (see Fig. 1). They found that the $\mathrm{T}$ dwarfs observed were generally faster rotators than the M stars in the sample. Assuming brown dwarfs are around $\sim 1 \mathrm{R}_{J u p}$ in size (a reasonable assumption, given electron degeneracy in their cores, Burrows et al. 2003), they find a maximum rotational period for L and T brown dwarfs of 12.5 hours. No similar study has been done to date for young brown dwarfs, but considerations from conservation of momentum as these objects form and collapse suggest their periods may be somewhat longer.

Recently, Snellen et al. (2014) measured a rotational velocity using VLT CRIRES for the 8-10 $\mathrm{M}_{\text {Jup }}$ planet $\beta$ Pic b (Lagrange et al. 2009; Lagrange et al. 2010). This is the first rotational velocity measured for any extrasolar planet. In fact, $\beta$ Pic b rotates faster than any of the planets in the Solar system with $v_{\text {rotation }}=25 \pm 3 \mathrm{~km} / \mathrm{s}^{-1}$. Using spectral modeling from broadband photometry to infer a radius of $1.65 \pm 0.06 R_{\text {jup }}$, this gives a rotational period of $\sim 8.1 \pm 1.0$ hours. Thus, at least one exoplanet is also a rapid rotator.

In the case of objects with spectral types $>\mathrm{L} 2$, the expected source of surface inhomogeneities is patchiness in cloud cover or even multiple cloud components, especially at the L/T spectral type transition. Marley et al. (2010) find that patchy cloud models best bridge the transition between the $\mathrm{L}$ and $\mathrm{T}$ spectral types, with entirely clear models best describing the later $\mathrm{T}$ spectral types and single component, entirely cloudy models best describing the earlier L spectral types (see Fig. 1). Observationally, Skemer et al. (2012) find that patchy cloud coverage may be required to model observations across a wide wavelength range.

Earlier spectral types (e.g., M type brown dwarfs in young star-forming regions such as Taurus) may have variability dominated by mechanisms other than clouds: e.g., accretion from a circum-brown-dwarf or circum-planetary disk or magnetic phenomena such as starspots. In contrast, $\mathrm{L}$ and $\mathrm{T}$ type brown dwarfs are too cool to have significant magnetically-driven starspots.

\section{Variability in Brown Dwarfs}

Variability likely due to cloud structure is already found in numerous $\mathrm{L}$ and $\mathrm{T}$ type field brown dwarfs. Indeed, for the closest known $\mathrm{L} / \mathrm{T}$ transition brown dwarf binary WISE J104915.57531906.1AB (generally referred to as Luhman 16AB, consisting of T0.5 and L7.5 components, Luhman 2013, Burgasser et al. 2013) resolved MPG/ESO $2.2 \mathrm{~m}$ GROND simultaneous six-band (r'i'z' JHK) photometric monitoring yielded detections of periodic variability in all 6 bands for Luhman 16B, with amplitudes from $5-15 \%$ (see Fig. 2, Biller et al. 2013, see also Gillon et al. 2013) and in i'z' for Luhman 16A with amplitudes from 1-3\% (Biller et al. 2013). This variability has a measured period of 4.9 hours, evolves on daily to weekly timescales (Gillon et al. 2013), and is likely due to changing cloud structures on these objects. Recent large-scale surveys of brown dwarf variability with Spitzer have revealed mid-IR variability of up to a few percent in $>50 \%$ of L and T type brown dwarfs (Metchev et al. 2015, see Fig. 3). (Buenzli et al. 2014) find that $\sim 30 \%$ of the L5-T6 objects surveyed in their HST SNAP survey show variability trends and large ground-based surveys also find ubiquitous variability (Radigan et al. 2014, 

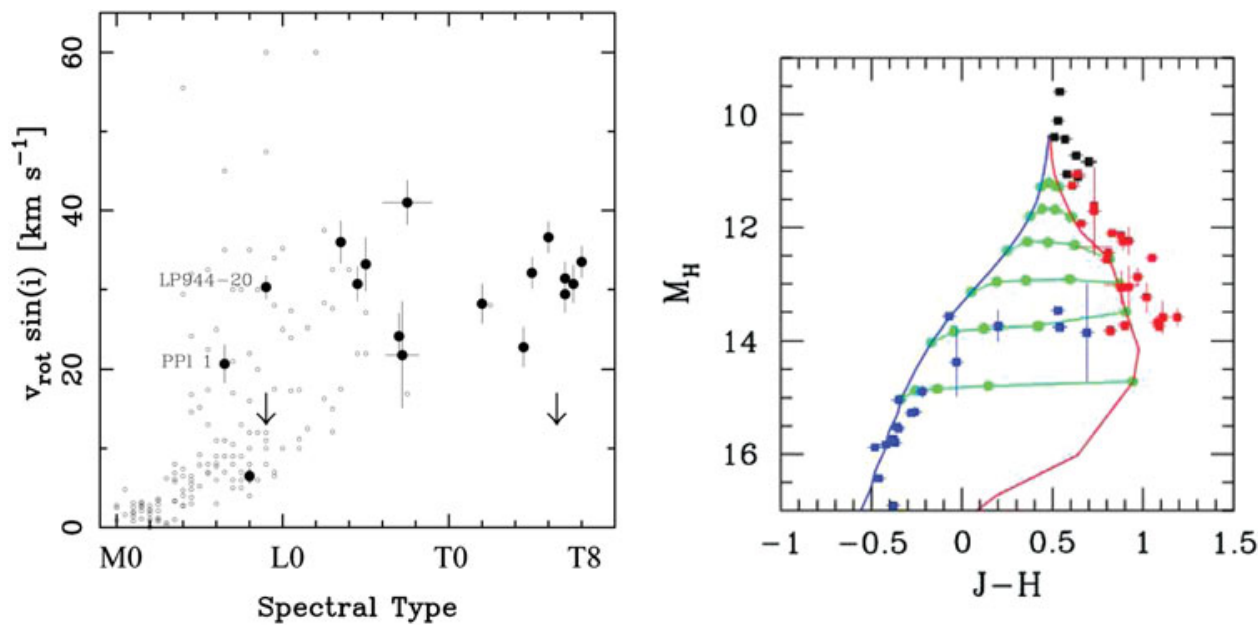

Figure 1. Left: Figure from (Zapatero Osorio et al. 2006). Keck NIRSPEC measurements of vsini for $19 \mathrm{M}$ to $\mathrm{T}$ objects show that T-type objects are generally faster rotators than M-type objects and set a maximum rotation period of 12.5 hours for the sample. Right: figure from (Marley et al. 2010). Black points are M stars, red points are L dwarfs, and blue points are T dwarfs. The solid red line is a single-component, completely cloudy model, the solid blue line is a completely clear model, and the solid green lines are patchy cloud models with different values of the cloud sedimentation parameter $\mathrm{f}_{\text {sed }}$. The patchy cloud models best reproduce the notable shift from red to blue colors seen at the L/T spectral type transition.
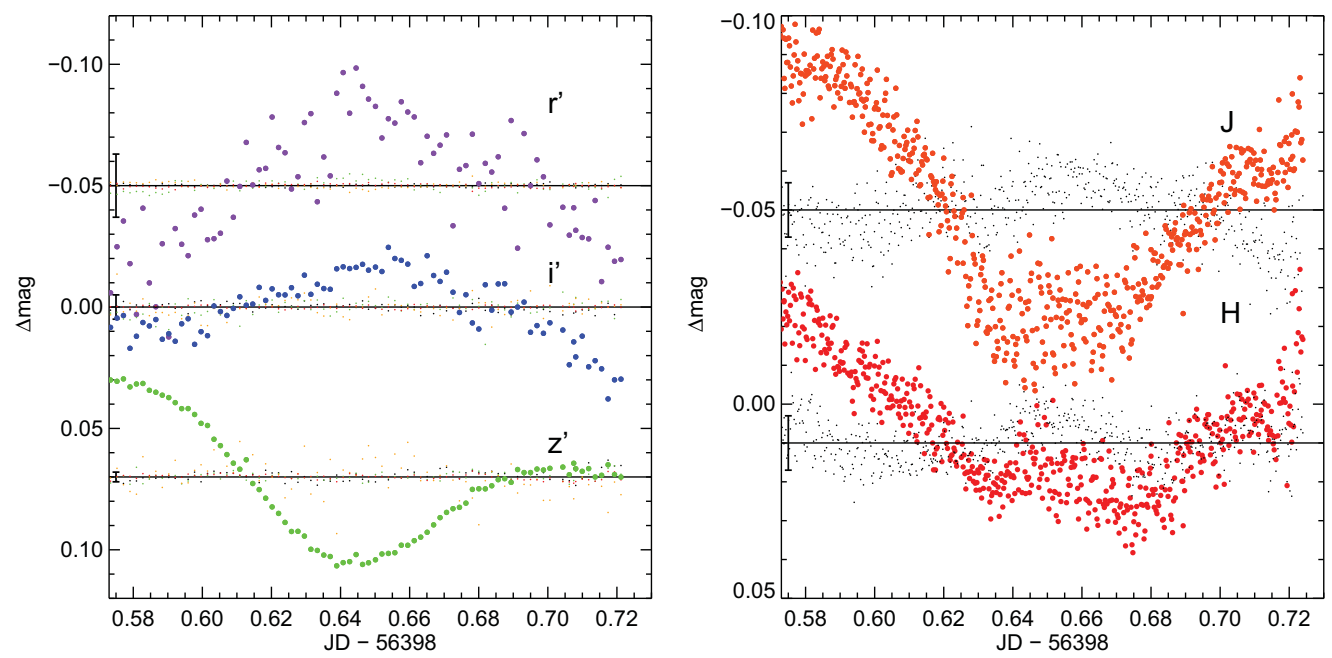

Figure 2. Figure from (Biller et al. 2013). Multiwavelength rizJHK $\mathrm{J}_{S}$ unresolved variability monitoring of the nearest brown dwarfs, the L/T transition binary Luhman $16 \mathrm{AB}$ using the GROND imager at the ESO/MPG $2.2 \mathrm{~m}$ telescope. Variability is seen from the few percent level up to the $15 \%$ level in all 6 bands.

Wilson et al. 2014, see Fig. 3). While variability amplitude may be increased across the $\mathrm{L} / \mathrm{T}$ transition (Radigan et al. 2014), variability is now robustly observed across the full range of $\mathrm{L}$ and $\mathrm{T}$ spectral types. Thus, variability may be expected for young extrasolar planets, which share similar $\mathrm{T}_{\text {eff }}$ and $\mathrm{L}$ and $\mathrm{T}$ spectral types. Indeed, the spectrum of the young planet HR $8799 \mathrm{~b}$ is nearly identical to that of the most variable brown dwarf known, 2M 2139 (27\% variable, Radigan et al. 2012). 

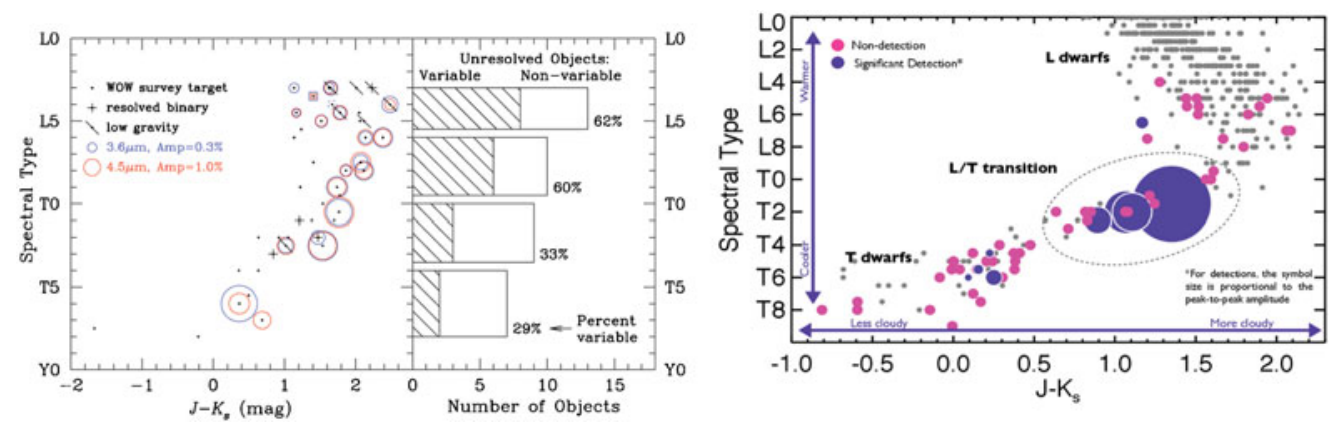

Figure 3. Left: Figure from Metchev et al. (2015), from a Spitzer mid-IR variability survey of 44 brown dwarfs. Metchev et al. (2015) find ubiquitous variability at low amplitudes as well as a tentative association ( $92 \%$ confidence) between low surface gravity and high-amplitude variability among L3L5.5 dwarfs. Right: Figure from Radigan et al. (2014), from a variability survey of 62 brown dwarfs. Of the 57 brown dwarfs included in their final analysis, Radigan et al. (2014) found that 9 were significantly variable with $>99 \%$ confidence, with higher variability amplitudes found for $\mathrm{L} / \mathrm{T}$ transition objects.

\section{Variability in Planetary Mass Objects}

Young directly imaged extrasolar giant planets and very low mass young brown dwarfs / planetary mass objects (estimated mass $<25 \mathrm{M}_{J}$ up using the conservative planet definition of Schneider et al. 2011, henceforth "young planetary mass objects" or PMOs) are in fact much better targets for variability monitoring (and hence, testing atmospheric models) than field $\mathrm{L}$ and $\mathrm{T}$ dwarfs. Field $\mathrm{L}$ and $\mathrm{T}$ dwarfs often have very uncertain distances and no age constraints, as these objects are degenerate with age - an old higher-mass object is indistinguishable from a young lower-mass object. In contrast, distances and ages for young exoplanets are well-measured. PMOs that are members of young clusters (1-10 Myr) or young moving groups (10-200 Myr) have well-defined ages. Extrasolar giant planets that are companions to stars often have well-defined distances, as their bright host stars generally have Hipparcos parallaxes. For free-floating young PMOs, cluster membership can provide a good distance estimate. Well-defined ages and distances translate to well-defined luminosities and stronger constraints on models.

The key physical difference between field brown dwarfs and young substellar objects / planetary companions $\left(<25 \mathrm{M}_{J u p}\right)$ is the lower surface gravity of the young, low mass objects relative to their older counterparts. Surface gravity plays an important role in the L-T spectral type transition; L-T transition appears to occur at lower temperatures for low-gravity exoplanets compared to higher gravity brown dwarfs (Marley et al. 2012). Surface gravity variations will likely affect cloud properties - variability studies are a direct probe of this.

\subsection{Variability in Free-floating Planetary Mass Objects}

While instruments such as GPI (Macintosh et al. 2014) and SPHERE (Beuzit et al. 2008) achieve contrasts suitable to enable variability studies for a handful of exoplanet companions, it is currently prohibitively difficult to study variability for a statistically significant sample of directly imaged planets within 1" of their star. However, we can circumvent the contrast issue and probe variability for young 3-25 $\mathrm{M}_{J \text { up }}$ objects by observing widely ( $>1$ ") separated companions and isolated very low mass young brown dwarfs / PMOs.

I (along with collaborators at Edinburgh, MPIA, IPAG, University of Hawaii, and elsewhere) am currently conducting the first statistically significant survey of weather 

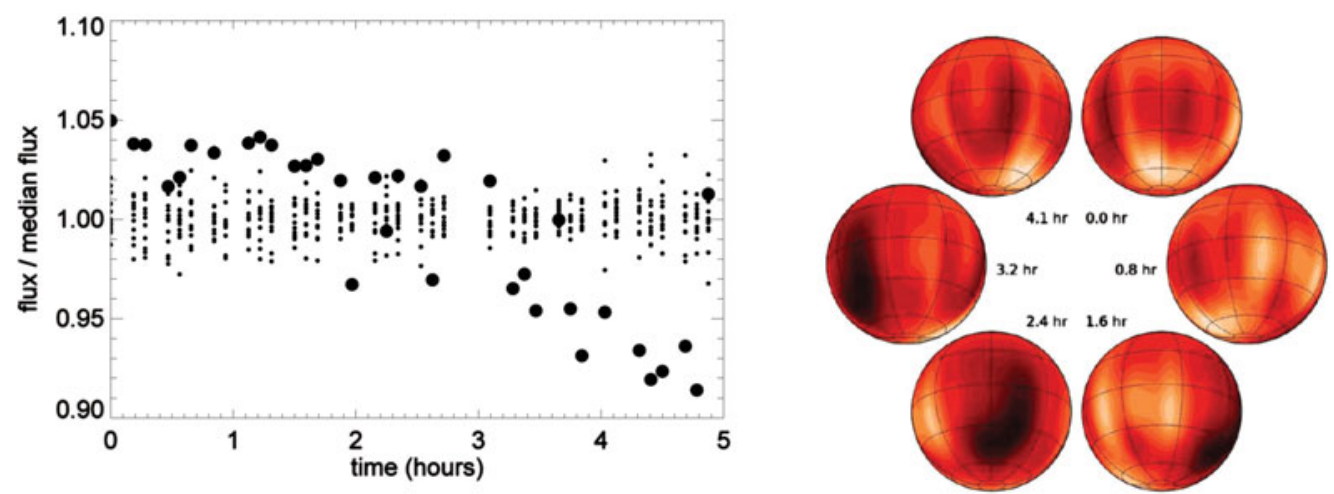

Figure 4. Left: First tentative detection from our SOFI survey of 22 young brown dwarfs and planetary mass objects. Lightcurves are shown after detrending using a calibration curve built from 8 carefully selected reference stars. The variable target is plotted with large filled circles; small circles show detrended lightcurves for the 8 reference stars. Comparing the peak-to-valley amplitude of the variability with the rms attained on similar brightness reference stars, variability is detected in this case at the 10- $\sigma$ level. Right: Figure from Crossfield et al. (2014). Doppler imaging map of the brown dwarf Luhman 16B. It will eventually be possible to make similar maps for exoplanet companions using high resolution spectrographs at extremely large telescopes, such as METIS, planned for the E-ELT.

patterns on young planetary mass objects using NTT SOFI. This project is the very first targetted survey of exoplanet variability. We have obtained to date $J$-band photometric variability monitoring observations for a sample of 22 young PMOs or very low mass brown dwarfs (mass estimates $<25 \mathrm{M}_{\text {Jup }}$ according to the conservative "planet" definition of Schneider et al. 2011) which are either known members of young star-forming clusters (age $<8$ Myr) or of nearby moving groups (age $<100 \mathrm{Myr}$ ). Our carefully chosen sample are all: 1) either high-probability cluster or moving group members with well-estimated ages and distances or with other indicators of young age (e.g., signs of low surface gravity), 2) with spectral type $\mathrm{L} 1$ or greater, e.g., predicted $\mathrm{T}_{\text {eff }}<2000 \mathrm{~K}$, and, 3) if a companion, with contrast low enough or at wide enough separation from its primary to easily be retrieved in a single SOFI exposure. The vast majority of our carefully selected sample were identified as young objects only recently, (see e.g., Gagné et al. 2014, 2015). We avoid earlier spectral types (e.g., M type brown dwarfs in young star-forming regions such as Taurus) as these objects may have variability dominated by mechanisms other than clouds: e.g., accretion from a circum-brown-dwarf or circum-planetary disk or magnetic phenomena such as starspots. In contrast, L and T type brown dwarfs are too cool to have significant magnetically-driven starspots. We also avoid field late $\mathrm{T}$ and $\mathrm{Y}$ brown dwarfs. While some of these objects likely have planetary masses, their ages are unconstrained, leading to large uncertainties on their mass estimates from models.

Buenzli et al. (2014) find that $\sim 30 \%$ of the L5-T6 objects surveyed in their HST SNAP survey show variability trends. For our 22 targets, we thus expect to find variability in up to $\sim 6$ of our targets in the most optimistic case that young, planetary mass objects share identical statistical properties to these older populations. Data analysis is still in progress; however, one tentative detection is presented in Fig. 4. Followup of tentative detections in our October / November 2014 data will take place in August 2015.

\subsection{Variability in Exoplanet Companions}

Searches for variability in exoplanet companions have only recently become possible with the advent of next generation planet imagers such as GPI and SPHERE. Planets which 
previously required $\sim 1$ hour of on-sky time to image can be detected even in 5-10 minutes of data from these imagers! Thus, multiple groups are pursuing variability studies for bright exoplanet companions such as $\beta$ Pic b and HR8799b. It is important to constrain variability of these objects now, as significant variability in exoplanet companions will lead to even more exciting followup possibilities with future extremely large telescope (ELT) instruments. For instance, Crossfield et al. (2014) used VLT CRIRES to produce a surface map of the highly variable $\mathrm{L} / \mathrm{T}$ transition brown dwarf Luhman 16B using the doppler imaging technique (see Fig. 4). This is the very first surface map of a brown dwarf - if young planets such as $\beta$ Pic b are similarly variable in the $\mathrm{L}^{\prime}$ bandpass, the high resolution spectroscopy mode anticipated for the E-ELT mid-IR instrument METIS will enable similar mapping of exoplanet surfaces (see e.g., Snellen et al. 2014).

\section{Conclusions}

The first surveys of variability in young free-floating PMOs as well as the first searches for variability in exoplanet companions are currently underway. In the next few years, these studies will provide a key probe of cloud properties in PMO atmospheres. This is already the case for field $\mathrm{L}$ and $\mathrm{T}$ brown dwarfs, where variability studies indicate that cloud inhomogeneity must be quite common for these objects.

\section{References}

Barman, T. S., Macintosh, B., Konopacky, Q. M., \& Marois, C. 2011, ApJ, 733, 65

Beuzit, J.-L., Feldt, M., Dohlen, K., et al. 2008, proc. SPIE, 7014,

Biller, B. A., Crossfield, I. J. M., Mancini, L., et al. 2013, ApJ Letters, 778, L10

Bowler, B. P., Liu, M. C., Dupuy, T. J., \& Cushing, M. C. 2010, ApJ, 723, 850

Buenzli, E., Apai, D., Radigan, J., Reid, I. N., \& Flateau, D. 2014, ApJ, 782, 77

Burgasser, A. J., Sheppard, S. S., \& Luhman, K. L. 2013, ApJ, 772, 129

Burrows, A., Sudarsky, D., \& Lunine, J. I. 2003, ApJ, 596, 587

Crossfield, I. J. M., Biller, B., Schlieder, J. E., et al. 2014, Nature, 505, 654

Gagné, J., Lafrenière, D., Doyon, R., Malo, L., \& Artigau, É. 2014, ApJ, 783, 121

Gagné, J., Lafrenière, D., Doyon, R., Malo, L., \& Artigau, É. 2015, ApJ, 798, 73

Gillon, M., Triaud, A. H. M. J., Jehin, E., et al. 2013, A\& A, 555, L5

Heinze, A. N., Metchev, S., Apai, D., et al. 2013, ApJ, 767, 173

Janson, M., Brandt, T. D., Kuzuhara, M., et al. 2013, ApJ letters, 778, L4

Kuzuhara, M., Tamura, M., Kudo, T., et al. 2013, ApJ, 774, 11

Lagrange, A.-M., Gratadour, D., Chauvin, G., et al. 2009, A\&SA, 493, L21

Lagrange, A.-M., Bonnefoy, M., Chauvin, G., et al. 2010, Science, 329, 57

Luhman, K. L. 2013, ApJ letters, 767, L1

Macintosh, B., Graham, J. R., Ingraham, P., et al. 2014, PNAS, 111, 12661

Marley, M. S., Saumon, D., \& Goldblatt, C. 2010, ApJ letters, 723, L117

Marley, M. S., Saumon, D., Cushing, M., et al. 2012, ApJ, 754, 135

Marois, C., Macintosh, B., Barman, T., et al. 2008, Science, 322, 1348

Marois, C., Zuckerman, B., Konopacky, Q. M., et al. 2010, Nature, 468, 1080

Metchev, S. A., Heinze, A., Apai, D., et al. 2015, ApJ, 799, 154

Radigan, J., Jayawardhana, R., Lafrenière, D., et al. 2012, ApJ, 750, 105

Radigan, J., Lafrenière, D., Jayawardhana, R., \& Artigau, E. 2014, ApJ, 793, 75

Rameau, J., Chauvin, G., Lagrange, A.-M., et al. 2013, A\&A, 553, A60

Rameau, J., Chauvin, G., Lagrange, A.-M., et al. 2013, arXiv:1305.7428

Schneider, J., Dedieu, C., Le Sidaner, P., Savalle, R., \& Zolotukhin, I. 2011, A\&A, 532, A79

Skemer, A. J., Hinz, P. M., Esposito, S., et al. 2012, ApJ, 753, 14

Snellen, I. A. G., Brandl, B. R., de Kok, R. J., et al. 2014, Nature, 509, 63

Wilson, P. A., Rajan, A., \& Patience, J. 2014, A\& A, 566, A111

Zapatero Osorio, M. R., Martín, E. L., Bouy, H., et al. 2006, ApJ, 647, 1405 